\title{
Peripheral Nerve Tumors as an Ongoing Challenge in Neuro-oncology: An Overview of Their Biological and Technical Nuances
}

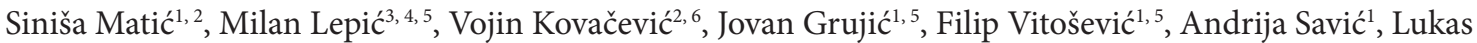 \\ Rasulićs, \\ ${ }^{1}$ Clinic for Neurosurgery, Clinical Center of Serbia, Belgrade, Serbia, ${ }^{2}$ Faculty of Medical Sciences, University of Kragujevac, \\ Kragujevac, Serbia, ${ }^{3}$ Faculty of Medicine of the Military Medical Academy, University of Defense, Belgrade, Serbia, ${ }^{4}$ Clinic for \\ Neurosurgery, Military Medical Academy, Belgrade, Serbia, ${ }^{5}$ Faculty of Medicine, University of Belgrade, Belgrade, Serbia, \\ ${ }^{6}$ Clinic for Neurosurgery, Clinical Center of Kragujevac, Kragujevac, Serbia
}

Correspondence: lukas.rasulic@gmail.com; Tel.: + 38163205 591; Fax.: + 381113615577

Received: 9 October 2020; Accepted: 7 December 2020

\begin{abstract}
This paper aims to provide an overview of recent advances in the diagnosis and treatment of peripheral nerve tumors (PNTs) with regard to biological and technological nuances, and to highlight some recommendations for achieving better outcomes in the treatment of patients suffering from PNT. PNTs are probably the most challenging entity in the field of peripheral nervous system surgery. The goal of removing a nerve tumor while also preserving nerve function at the same time is often complicated, regardless of the surgeon's experience. Still, in most cases, high-quality results can be achieved upon carefully planned surgery. Clinical presentation, diagnosis, and indications for a specific type of treatment of PNTs still remain a topic of debate. Recent technological advances have led to an exponential improvement in the field with utilization of intraoperative ultrasound, neurostimulation devices, and intraoperative electrophysiological monitoring, along with the development of modern surgical techniques, whereby a multidisciplinary and individually shaped approach is necessary. Conclusion. These advances, however, still remain limited, and recent research is focused on the development of biological therapy. Biologically targeted therapies will emerge when there is a better understanding of the genetic and molecular mechanisms driving the development and growth of PNTs.
\end{abstract}

Key Words: Peripheral Nerve - Tumor • Surgery • Biology • Technology.

\section{Introduction}

Peripheral nerve tumors (PNTs) are probably the most challenging entity in the field of peripheral nervous system surgery. The goal of removing the nerve tumor while simultaneously preserving nerve function is sometimes extremely complicated, despite the surgeon's experience. Still, in most cases, high-quality results can be achieved upon carefully planned surgery (1). Different types of tumors require different surgical and conservative treatment strategies; therefore, a good working knowledge of anatomy and pathophysiology of the various tumors is mandatory, as well as familiarity with the modern surgical techniques (2).
Recent technological advances have led to an exponential improvement in the field of PNT treatment with the utilization of intraoperative ultrasound, neurostimulation devices, and intraoperative electrophysiological monitoring, as well as with the development of the modern surgical techniques. Multidisciplinary cooperation among the neurosurgeon, orthopedic surgeon, vascular surgeon, plastic surgeon, physiatrist, physiologist, neurologist, and radiologist is essential for further development of PNT treatment. Nevertheless, these technological advances still remain limited, and recent research is now focused on the development of biological therapy (3). 
This paper aims to provide an overview of recent advances in PNT diagnosis and treatment with regard to biological and technological nuances, and to highlight some recommendations for achieving better outcomes in the treatment of patients suffering from PNTs.

\section{General Characteristics and Classification}

The clinical presentation of PNT is commonly asymptomatic, but typical presenting signs (or a combination of signs) include a palpable mass adjacent to the nerve and nerve palsy with or without pain. Nonspecific symptoms related to mass effect are components of a clinical presentation in symptomatic patients, but it is important to have an understanding about the nature of the tumor before surgery. The diagnostic assessment comprises of the patient's history and a clinical evaluation, followed by neuroradiological imaging and electrophysiological studies. Most commonly, the histological origin of these tumors is from the nerve sheath, but they can also be derived from neural structures (4) (Table 1).

Table 1. World Health Organization Classification of Nervesheath Tumors

\begin{tabular}{ll}
\hline Benign & Malignant \\
\hline $\begin{array}{l}\text { Schwannoma (including } \\
\text { variants) }\end{array}$ & $\begin{array}{l}\text { Malignant peripheral nerve } \\
\text { sheath tumor }\end{array}$ \\
\hline Melanotic schwannoma & $\begin{array}{l}\text { Epithelioid malignant nerve } \\
\text { sheath tumor }\end{array}$ \\
\hline $\begin{array}{l}\text { Neurofibroma (including } \\
\text { variants) }\end{array}$ & Malignant Triton tumor \\
\hline Plexiform neurofibroma & Malignant granular cell tumor \\
\hline Perineurioma & Ectomesenchymoma \\
\hline Malignant perineuriomas & - \\
\hline Granular cell tumor & - \\
\hline $\begin{array}{l}\text { Dermal nerve sheath } \\
\text { myxoma }\end{array}$ & - \\
\hline Solitary circumscribed \\
neuroma
\end{tabular}

Benign PNT lesions are much more common than malignant lesions. Benign peripheral nerve sheath tumors (BPNSTs) represent approximately $1 \%$ of all soft tissue tumors. While they are more frequently found in the upper limbs, mostly affecting large nerve trunks, any nerve can ultimately be affected. BPNSTs most often include schwannomas and neurofibromas. They can appear spontaneously or within different syndromes (neurofibromatosis-1 and 2 [NF1 and NF2], Schwannomatosis, etc.) $(5,6)$. Benign tumors and tumor-like lesions of nonneural origin are rare lesions (usually ganglion cysts, vascular tissue or desmoid tumors), and were sporadically reported within the nerves (7-11). Hemangioblastomas of peripheral nerve were also reported (12).

Malignant peripheral nerve sheath tumors (MPNSTs) are aggressive, soft-tissue sarcomas that originate from nerve structures. Although these tumors are rare, they are clinically challenging to treat with a 5-year survival of 50\% (13). Patients with NF1 and previous radiation exposure are at highest risk for the development of MPNST.

Current evidence suggests that in most cases, benign and malignant lesions should be differentiated pre-operatively based on clinical and radiographic characteristics (14). In malignant tumors, loss of function and pain are far more common and neurological deficit is usually more severe. Some degree of pain is present in about $75 \%$ of all patients with PNTs. Positional pain or pain induced by pressure (Tinel's sign) are common in both; pain at rest is a far more specific symptom for MPNST. A possible explanation for this relationship is due to the chemical nature of pain because of the release of mediators $(\mathrm{H}+$, proteolytic enzymes, cytokines, GF, etc.) by malignant cells that stimulate chemoreceptive pain fibers. Additionally, an invasion of the perineurium is necessary for the development of pain at rest (15). Rapid mass enlargement is also strong predictor for malignancy. Nevertheless, this preoperative differentiation is not always possible, and many of the tumors remain indistinct even after resection (Figure 1).

Overall, surgery is successful without function impairment in $90 \%$ of BPNST, and about $10 \%$ 


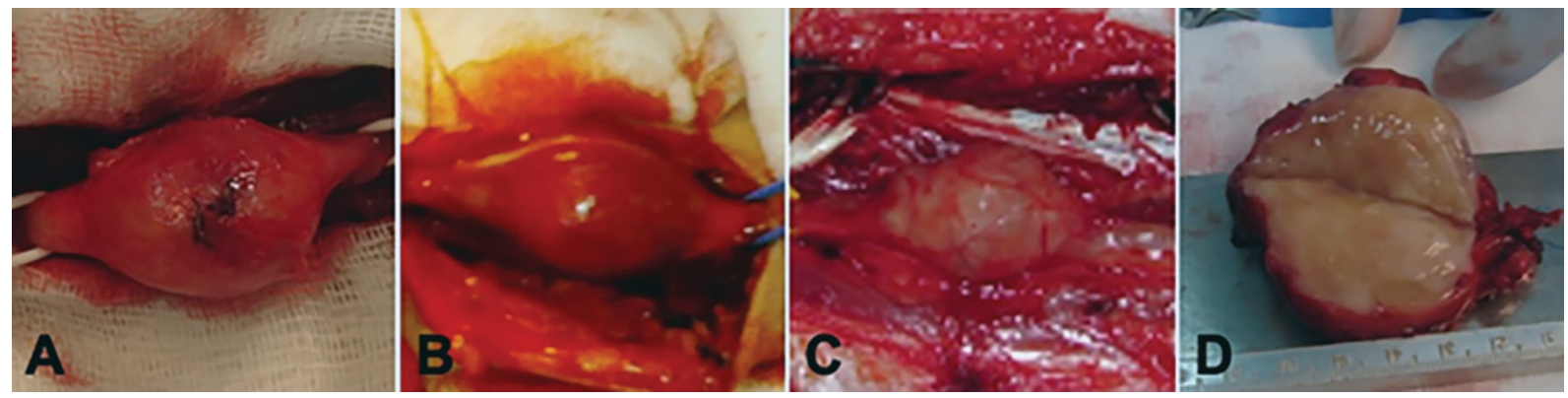

Figure 1. Intraoperatively indistinct morphologic macroscopic appearance of the solitary tumors of the peripheral nerves: (A) radial nerve Schwannoma; (B) ulnar nerve hemangioblastoma; and (C) tibial nerve MPNST within the nerve and (D) extirped and transected to find the internal structure without any signs of malignancy (homogenous solid tumor tissue).

of patients presented with post-operative motor weakness (6). Patients with larger tumor size have a higher risk for developing neurological deficit (16). The majority of postoperative neurological symptoms are transient and do not lead to permanent disability, but they can be a problem when considering patient satisfaction with the surgery. The cause of postoperative neurological deficit in BPNST remains unclear, although several mechanisms have been proposed, like tumor compression, iatrogenic direct lesion during surgery, or ischemia of the nerve associated with the surgery (17). Takase et al. have the view that damaging the fascicles around the tumor during extracapsular excision was one of the main causes of new postoperative neurological deficits (18).

\section{Nuances in the Evaluation and Management of Peripheral Nerve Tumors}

Complex biological nuances are related to the specific features of the PNT, and these are strongly interrelated with numerous controversial points considered as technical nuances at every stage of the assessment and treatment of these patients.

\section{Imaging}

\section{Magnetic Resonance Imaging}

Magnetic resonance imaging (MRI) is the main imaging modality for diagnosis, evaluation, and treatment planning. Clinical and imaging characteristics can point toward the diagnosis of a benign or malignant tumor. The boundary between the tumor and the nerve is sometimes difficult to determine because the nerve may be distended and stretched over the mass. MRI evaluation consists of different sequences (e.g., T1, T2, FAT SAT, and IV Gadolinium), with images in the same plane (usually axial) supplemented by at least one other sequence in an orthogonal (i.e., sagittal or coronal) plane (19).

Positioning the patient and area for exploration at the magnet center is essential for ensuring high quality images. These can be further improved by contrast enhancement, 3D or 4D images, tractography, or by magnetic resonance angiography (MRA), depending on the type of lesion. Gadolinium-enhanced images must always be obtained to provide conclusive differentiation between a cystic and a solid lesion. A normal nerve has an intermediate signal intensity that is identical to muscle on $\mathrm{T} 1$ and $\mathrm{T} 2$ sequences. It is best viewed on $\mathrm{T} 1$ sequences in fatty layers or on T2-weighted fast spin-echo and T2 fat-suppressed sequences in which the nerve appears hyperintense compared to muscle. There is no nerve enhancement after contrast injection. When dealing with a mass located in the region of a nerve, MRI allows for a diagnosis in $75 \%$ of cases. Coronal and sagittal images provide individualization of the nerve's entry and exit from the tumor.

Figure 2 depicts the issue of indistinct features in MRI. 

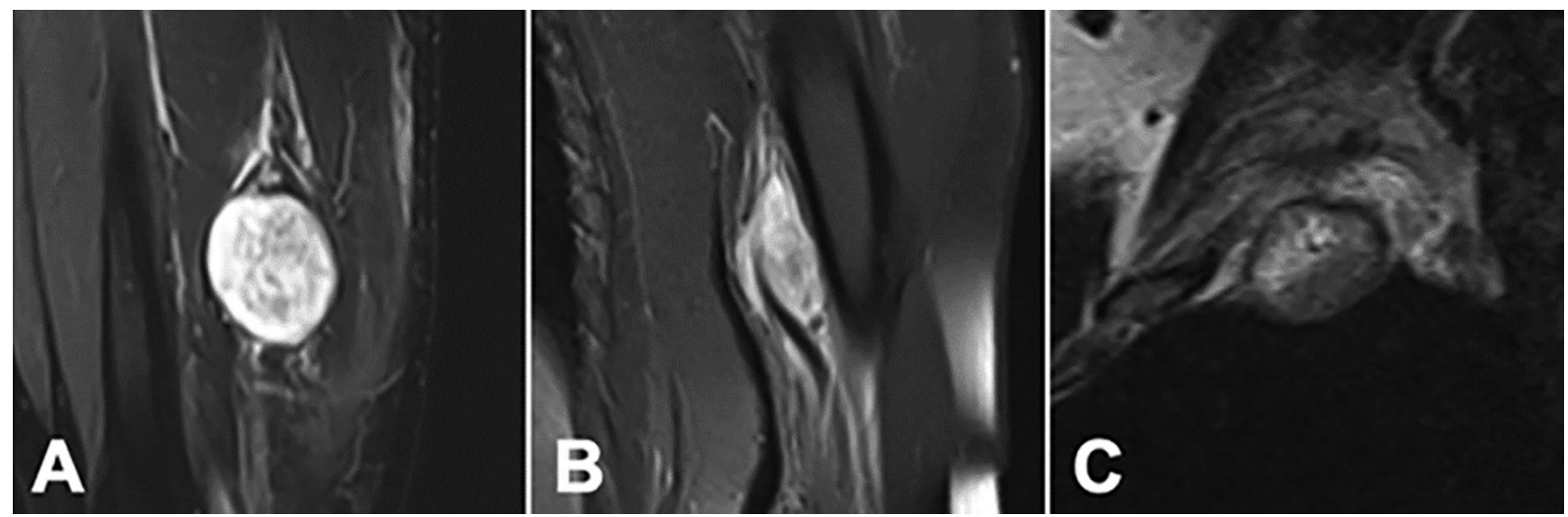

Figure 2. Indistinct features of benign and malignant peripheral nerve tumors on contrast enhanced MRI: (A) tibial nerve Schwannoma in the proximal part of the lower leg; (B) median nerve MPNST in the forearm; and (C) brachial plexus MPNST as seen on MRI without contrast. Note the homogenous structure and similar contrast enhancement of both benign and malignant PNTs.

\section{Ultrasound}

Ultrasound (US) is the imaging modality of choice for the diagnosis of BPNST. As a general rule, peripheral nerves are more visible when surrounded by the tissues with different echo features. Peripheral nerves appear as tubular structures made of hypo-echoic nerve fascicles embedded in a hyperechoic connective tissue esponding to the epineurium. Longitudinally, US images present a fascicular pattern, and transversely, fascicles appear rounded or oval in shape, giving the nerve its typical honeycomb appearance. US diagnosis of a nerve tumor is based on the existence of a mass in continuity with the nerve at its proximal and distal poles (14).

One drawback of US is the fact that differentiation between lymphadenopathy, neuroma, or liquid tumor (hematoma, abscess, or thrombosed aneurysm), is more operator-dependent than MRI, and US imaging of nerve lesions is rarely specific. US cannot replace MRI for determining the topography, the exact extent of tumor formation, or the presence of invasion. However, its features enable the physician to analyze the entire nerve course, evaluate vascularization in real time, and look for other remote tumors. Additionally, US is increasingly used intraoperatively (20).

\section{Computerized Tomography and Radiography}

Computerized tomography (CT) is rarely performed because of its low resolution for soft tis- sues. It shows the nerve only as a non-specific structure of intermediate density. CT is a complementary tool and may be helpful in visualizing an underlying bone abnormality (21).

\section{Indication for Surgical Treatment}

For tumors with a benign appearance, indications for surgery include pain, neurological deficit, and local mass effect. Relative indications are patient motivation and aesthetic effect. Regular follow up is acceptable for small tumors with benign appearance and a minimum of symptoms; however, this asymptomatic state is temporary and will progressively develop into functional impairment as the tumor size increases (16). Also, neurosurgeons should remember that surgery of smaller benign tumors is technically easier and will prevent further functional damage (1).

\section{Fascicular Invasion}

Because of the intimate relationship between functioning nerve fascicles and tumor tissue, these lesions should undergo careful surgical resection during surgery. The main characteristic of Schwannoma is the displacement of fascicles rather than their infiltration, with the exception of the origin fascicle from which they arise $(22,23)$. Neurofibromas present as more diffuse expansions 
of the nerve than schwannomas. They may have single or multiple fascicles that enter and leave the nerve. In the majority of cases, the nerve of origin is nonfunctional at the time a diagnosis is established. Plexiform neurofibromas have a predominant interfascicular histologic growth pattern with redundant loops of expanded nerve fascicles. The presence of axons within the tumor helps to distinguish a neurofibroma from a schwannoma.

When possible, the main treatment goal should be complete tumor resection with preservation of nerve function. The surgeon is usually able to perform gross total marginal resection in cases of benign PNSTs. For Schwannomas, gross total resection starts by incising the capsule surrounding the tumor, enucleating the tumor mass, and sectioning the parent nerve fascicle that gave rise to the tumor under magnification. If the capsule is firmly adherent to the parent nerve, it should be partially left behind (23). Avoiding damage to the surrounding fascicles is mandatory, which will result in the resolution of symptoms and preservation of function in the majority of cases (22). For benign PNT, especially of the plexiform type, dissection of the tumor and nearby nerve must be undertaken with extreme care. Intraoperative neurophysiologic monitoring plays a considerably important role during surgery for plexiform Schwannomas or neurofibromas to determine whether the nerve associated with the tumor has motor function. If electrical stimulation of a nerve fascicle adjacent to tumor produces a motor response at $1 \mathrm{~mA}$, the fascicle should not be sacrificed.

Recently, Stone and Spinner proposed a "Go for the Gold" technique for benign peripheral nerve sheath tumors (24). This technique favors preservation of all functional fascicles through the identification of all pseudo capsule layers before removing of the mass with the true capsule, leaving all en passant functioning nerve fascicles and epineurial vessels preserved.

\section{Intraoperative Neuromonitoring}

Intraoperative neuromonitoring (IONM) is a proven and useful tool in neurosurgery, but its usage in PNT surgery only came years after brain surgery. Main purpose for using IONM is to find a safe entry zone for intracapsular enucleation of a schwannoma since the tumor can be surrounded by a bundle of nerve fascicles $(16,25)$. Direct nerve stimulation (DNS) is, therefore, especially useful. Another IONM modality is utilization of nerve action potentials (NAPs), which is an electrical potential recorded from an exposed nerve in response to its electrical stimulation. NAPs allow for intraoperative monitoring of the functional integrity of peripheral nerve during peripheral nerve tumor surgery (26).

Motor evoked potentials (MEPs) alone is not able to predict postoperative loss of motor function, so usage of MEPs alone is not recommended. However, use of MEPs combined with free-run electromyography or direct nerve stimulation may improve the accuracy of nerve monitoring (17).

\section{Fluorescein}

Sodium fluorescein has been used in medicine for several decades, but its use in neurosurgery is recent. It was first used in brain tumor surgery 20 years ago, while the possible implications for its use in PNT surgery had been suggested only recently. Precise usage in terms of timing and dosage is still controversial, but some authors found sodium fluorescein useful for better identification of tumor tissue and distinguishing it from intact fascicles $(20,27)$. Another potential important usage is to identify residual tumor when resection is performed in a piecemeal fashion (28). Sodium fluorescein has been tested only for resection of Schwannoma so far, and further studies are needed to determine precise dose and timing of application for other PNTs.

\section{Indications for Functional Sacrifice}

Neurologic complications can occur after excision, including pain and loss of motor or sensory function $(22,29)$, even when a complete resection is achieved and adjacent fascicles were preserved. Complete (or en bloc) peripheral nerve tumor 

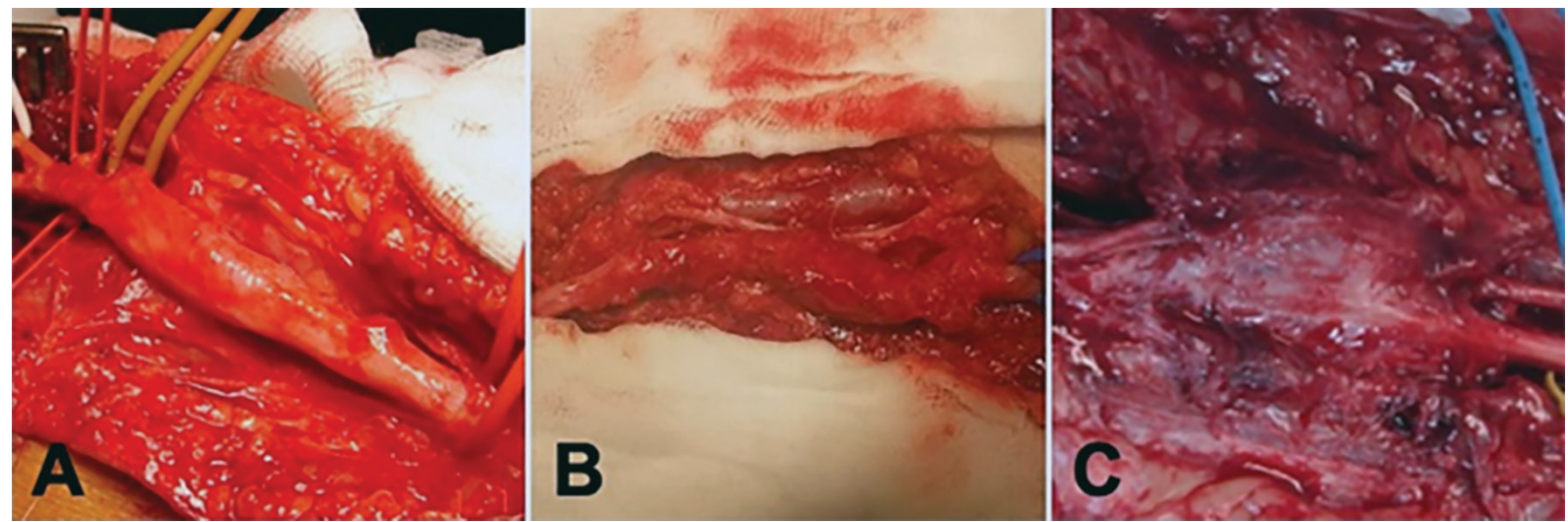

Figure 3. Intraoperatively indistinct fusiform lesions of the peripheral nerves: (A) Peroneal nerve intraneural ganglion cyst; (B) Median nerve neurofibroma; and (C) Median nerve MPNST within the soft tissues of the arm.

resection is only indicated for malignant or premalignant cases and implies resection of the entire segment of the affected peripheral nerve stem, including up to $2 \mathrm{~cm}$ of healthy nerve, with conscious functional sacrifice. In general, treatment decisions must consider whether or not the mass is symptomatic and has a high risk of malignancy. Surgical excision is the safest option in cases of symptomatic or asymptomatic growing PNTs. Operative techniques for the excision of probable benign PNTs should consider mass location, size, and the possibility of malignancy. Microsurgical technique and intraoperative identification of functional nerve fibers with electrical stimulation are always mandatory. Complete surgical resection without damaging functional fascicles is the goal. Most patients report significant pain relief and improvement of sensory motor deficits after surgery. Nevertheless, developing or worsening pain and neurological deficits can occur at follow-up. Recurrence rates may vary according to different types of benign PNTs and predisposing conditions. Figure 3 presents the similar appearance of the 3 completely different tumor classes.

In cases of neurological deficit and loss of function, reconstruction is mandatory (except in cases of MPNST). Reconstruction of the nervous stem in the same surgery is the best option and includes nerve grafting, nerve transfer, or other procedures for certain nerve types in a second stage surgery, such as tendon transfer. It was also reported that early radiotherapy may be applied without reinnervation process compromise; however, this remains controversial (30).

\section{Biopsy}

Soft tissue tumors in general may represent a diagnostic challenge, which is why diagnostic biopsy is performed. For PNTs, the role of biopsy is still controversial. Biopsy can be performed by needle, with or without guidance (CT or US), or in an open fashion. A needle biopsy can be done by a surgeon or radiologist. The main drawback of needle biopsy is risk of injuring intact nerve fascicles in the needle trajectory (29), which is not always avoided, even when US guidance is used (31). Another problem is risk of inconclusive sample for pathohistological analysis due to cellular pleomorphism (32).

Biopsy for benign PNTs is associated with an unacceptable risk of neurological deficit and is associated with a higher risk of postoperative deficits $(23,33)$. Higher postoperative deficits in previously biopsied tumors is explained by scar-tissue forming, which makes the dissection plane less clear (33). While keeping these criticisms in mind, biopsy is still recommended in cases with highly suspected malignancy $(23,34)$. The avoidance of biopsy is recommended in cases of expected benign pathology (22). Nevertheless, some authors still recommend biopsy for selected cases (35). 


\section{Management of MPNST}

Surgical treatment for malignant tumors includes radical resection, followed by adjuvant treatment modalities, such as radiotherapy and chemotherapy. In some cases, radical resection means amputation of any extremities with disarticulation. Chemotherapy agents used in MPNST treatment include erlotinib, sorafenib, imatinib, dasatinib, alisertib, ganetespib/sirolimus, bevacizumab/ RAD001, pexidartinib/sirolimus, and selumetinib/ sirolimus. MPNST is nevertheless a relatively chemoresistant tumor. Evidence suggests that in partially resected or unresectable tumors, chemotherapy produces regression in less than $33 \%$ of cases. NF1 patients also have a lower response rate to chemotherapy than sporadic cases- $17.6 \%$ compared to $55.3 \%$, respectively (36).

Radiotherapy provides local control and may delay the onset of recurrence, but it has little effect on long-term survival and should be given whenever possible for intermediate to high-grade lesions, and for low-grade tumors after a marginal excision. Postoperative radiotherapy involves irradiation of the entire operative field with a $5 \mathrm{~cm}$ field margin, and preoperative radiotherapy involves irradiation of the overt tumor alone again with a $5 \mathrm{~cm}$ margin (37).

Overall, the side effects of MPNST management include secondary malignancies, dermatitis, cellulitis, fibrosis, and risk of relapse due to failure to control local or metastatic disease. Complete resection can lead to local deformities and a risk of damage to critical internal structures (36). Overall prognosis is poor: the 5 -year rate of survival ranges from $15 \%$ to $50 \%$ (35). Metastases are extremely rare, and may include lungs, pleura, the breast, or skin, among other localizations. Chemotherapeutic treatment is emerging, although with only limited success - the overall survival is only $40 \%$ within 1 year of diagnosing metastatic MPNST (38).

\section{Biological Therapy}

A more thorough understanding of the genomic, epigenetic, signaling pathway, and microenvironment changes during the plexiform neurofibroma-
to-MPNST malignant transformation has offered hope for the development of more effective diagnostic and therapeutic strategies for MPNST. Recent clinical trials conducted in patients with NF1 for the treatment of symptomatic plexiform neurofibromas using inhibitors of the mitogen-activated protein kinase (MEK) have shown very promising results (39). Unfortunately, MEK inhibitors do not work in all patients and have significant side effects. In addition, preliminary evidence suggests single agent use of MEK inhibitors for MPNST treatment will fail, which is why it is important to find targets other than MEK for treatment of MPNST.

\section{Conclusion}

Peripheral nerve tumors can be extremely challenging lesions to manage. Magnification, use of intraoperative recordings, and knowledge of the gross and microscopic pathology are important to the surgeon undertaking tumor resection. An understanding of the tumor's disposition, coupled with an awareness of the fascicles that may be spared, can improve the surgeon's ability to resect benign nerve sheath tumors. The clinical presentation, diagnosis, and indications for a specific type of treatment for PNTs still remain a topic of debate. However, all collected data show that both an individual and multidisciplinary approach is necessary in the treatment of these tumors. Targeted therapies will emerge when there is a better understanding of the genetic and molecular mechanisms driving the development and growth of PNTs. Early referral and evaluation by a surgical specialist with expertise in peripheral nerve tumors can mitigate negative long-term sequelae.

Conflicts of Interest: The authors declare that they have no conflicts of interest.

\section{References}

1. Rasulic L. Current Concept in Adult Peripheral Nerve and Brachial Plexus Surgery. J Brachial Plex Peripher Nerve Inj. 2017;12(1):e7-14.

2. Martinez AP, Fritchie KJ. Update on Peripheral Nerve Sheath Tumors. Surg Pathol Clin. 2019;12(1):1-19. 
3. Rasulić L, Lepić M, Savić A, Lepić T, Samardžić M. Peripheral nervous system surgery: Travelling through no man's land to new horizons. Neurol India. 2019;67(Supplement):S9-15.

4. Fletcher CDM, World Health Organization, International Agency for Research on Cancer. WHO classification of tumours of soft tissue and bone. 4 th ed. Lyon: IARC Press; 2013. p. 468.

5. Milad M, Said D, Melisa E, Darya A, Babapour B. Surgical management of tibial nerve schwannoma under (intraoperative neurophysiologic monitoring. J Neurol Stroke. 2018;8(5):267-8.

6. Bharamagoudar PC, Marajakke S. Surgical Removal of Peripheral Nerve Schwannoma with Intraoperative Neurophysiological Monitoring. J Sci Soc. 2019;46:67-9.

7. Woertler K. Tumors and tumor-like lesions of peripheral nerves. Semin Musculoskelet Radiol. 2010;14(5):547-58.

8. Desy NM, Wang H, Elshiekh MAI, Tanaka S, Choi TW, Howe BM, et al. Intraneural ganglion cysts: a systematic review and reinterpretation of the world's literature. J Neurosurg. 2016;125(3):615-30.

9. Kim DH, Murovic JA, Tiel RL, Moes G, Kline DG. A series of 397 peripheral neural sheath tumors: 30-year experience at Louisiana State University Health Sciences Center. J Neurosurg. 2005;102(2):246-55.

10. Siqueira MG, Tavares PL, Martins RS, Heise CO, Foroni LH, Bordalo M, et al. Management of desmoid-type fibromatosis involving peripheral nerves. Arq Neuropsiquiatr. 2012;70(7):514-9.

11. Ferraresi S, Garozzo D, Bianchini E. Aggressive fibromatosis (desmoid tumor) of the radial nerve: favorable resolution. Case report. J Neurosurg. 2001;95(2):332-3.

12. Rasulic L, Samardzic M, Bascarevic V, Micovic M, Cvrkota I, Zivkovic B. A rare case of peripheral nerve hemangioblastoma-case report and literature review. Neurosurg Rev. 2015;38(1):205-9; discussion 9.

13. Porter DE, Prasad V, Foster L, Dall GF, Birch R, Grimer RJ. Survival in Malignant Peripheral Nerve Sheath Tumours: A Comparison between Sporadic and Neurofibromatosis Type 1-Associated Tumours. Sarcoma. 2009;2009:756395.

14. Chick G, Hollevoet N, Victor J, Bianchi S. The role of imaging in isolated benign peripheral nerve tumors: A practical review for surgeons. Hand Surg Rehabil. 2016;35(5):320-9.

15. Bakst R, Wong R. Mechanisms of Perineural Invasion. Journal of Neurological Surgery Part B: Skull Base. 2016;77(02):096-106.

16. Li X, Zhong G, Xu X, Wang K, Zhu Y, Qi X, et al. Surgical strategies for peripheral nerve schwannoma based on the intraoperative neurophysiological monitoring. Laparoscopic, Endoscopic and Robotic Surgery. 2019;2(3):65-9

17. Sasaki H, Nagano S, Yokouchi M, Setoguchi T, Shimada $\mathrm{H}$, Yamamoto $\mathrm{T}$, et al. Utility of intraoperative monitoring with motor-evoked potential during the surgical enucleation of peripheral nerve schwannoma. Oncol Lett. 2018;15(6):9327-32.

18. Takase K, Yamamoto K, Imakiire A. Clinical pathology and therapeutic results of neurilemmoma in the upper extremity. J Orthop Surg (Hong Kong). 2004;12(2):222-5.

19. Ahlawat S, Chhabra A, Blakely J. Magnetic resonance neurography of peripheral nerve tumors and tumorlike conditions. Neuroimaging Clin N Am. 2014;24(1):17192.

20. Pedro MT, Antoniadis G, Scheuerle A, Pham M, Wirtz CR, Koenig RW. Intraoperative high-resolution ultrasound and contrast-enhanced ultrasound of peripheral nerve tumors and tumorlike lesions. Neurosurg Focus. 2015;39(3):E5.

21. Pilavaki M, Chourmouzi D, Kiziridou A, Skordalaki A, Zarampoukas T, Drevelengas A. Imaging of peripheral nerve sheath tumors with pathologic correlation: pictorial review. Eur J Radiol. 2004;52(3):229-39.

22. Albert P, Patel J, Badawy K, Weissinger W, Brenner M, Bourhill I, et al. Peripheral Nerve Schwannoma: A Review of Varying Clinical Presentations and Imaging Findings. J Foot Ankle Surg. 2017;56(3):632-7.

23. Temple HT, Qadir R, Cuartas E, Ross AL, Levi AD. The Surgical Management of Symptomatic Peripheral Nerve Sheath Tumors. Neurosurgery. 2010;66(4):833-40.

24. Stone JJ, Spinner RJ. Go for the Gold: A "Plane" and Simple Technique for Resecting Benign Peripheral Nerve Sheath Tumors. Oper Neurosurg (Hagerstown). 2020;18(1):60-8.

25. Kim D-h, Choi J-g, Son B-c. Identification of Safe Zone with Intraoperative Neurophysiological Monitoring during Surgical Removal of Peripheral Nerve Tumor. The Nerve. 2016;2(2):78-80.

26. Saponaro-Gonzalez A, Perez-Lorensu PJ. Novel approach to continuous neurophysiological monitoring during surgery of peripheral nerve tumors. Surg Neurol Int. 2017;8:184.

27. Vetrano IG, Nazzi V, Acerbi F. What is the advantage of using sodium fluorescein during resection of peripheral nerve tumors? Acta Neurochir (Wien). 2020;162(5):11535.

28. Vetrano IG, Saletti V, Nazzi V. Fluorescein-guided resection of plexiform neurofibromas: how I do it. Acta Neurochir (Wien). 2019;161(10):2141-5.

29. Vucemilo L, Lajtman Z, Mihalj J, Plascak J, Mahovic Lakusic D, Muzinic D. Brachial Plexus Schwannoma - Case Report and Literature Review. Acta Clin Croat. 2018;57(2):366-71.

30. Liodaki E, Robiller S, Wenzel E, Mailaender P, Stang F. Novel Treatment of a Malignant Peripheral Nerve Sheath Tumor of the Median Nerve. Plastic and Reconstructive Surgery - Global Open. 2018;6(12):e2011.

31. Levi AD, Cajigas I, Debs L, Shelby Burks S, Perez-Roman RJ. The Risk of Peripheral Nerve Tumor Biopsy in Suspected Benign Etiologies. Neurosurgery. 2020;86(3):E326-32. 
32. Mir R, Singh VP, Kaul S. Varied presentation of schwannoma - a case study. Case Rep Oncol. 2010;3(3):354-61.

33. Perez R, Chen JM, Nedzelski JM. Intratemporal Facial Nerve Schwannoma: A Management Dilemma. Otol Neurotol. 2005;26(1):121-6.

34. Khu KJ, Midha R. Malignant Peripheral Nerve Sheath Tumors. World Neurosurg. 2016;94:566-7.

35. Strike SA, Puhaindran ME. Tumors of the Hand and the Wrist. JBJS Rev. 2020;8(6):e0141.
36. Durbin AD, Ki DH, He S, Look AT. Malignant Peripheral Nerve Sheath Tumors. Adv Exp Med Biol. 2016;916:495530 .

37. Perrin RG, Guha A. Malignant peripheral nerve sheath tumors. Neurosurg Clin N Am. 2004;15(2):203-16.

38. Hirbe AC, Rathore R, Ball T, Prudner BC. Diagnosis and management of malignant peripheral nerve sheath tumors: Current practice and future perspectives. Neurooncol Adv. 2020;2(Suppl 1):i40-9.

39. Killock D. Selumetinib benefits children with inoperable plexiform neurofibromas. Nat Rev Clin Oncol. 2020;17(5):273. 